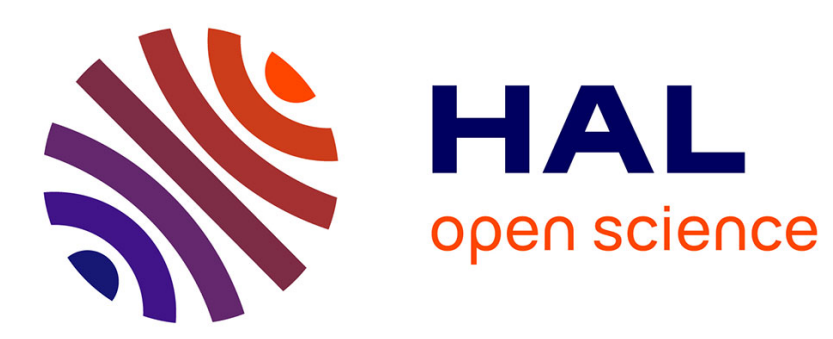

\title{
Automatic extraction of approximate repetitions in polyphonic MIDI files based on perceptive criteria
}

Benoît Meudic, Emmanuel Saint-James

\section{To cite this version:}

Benoît Meudic, Emmanuel Saint-James. Automatic extraction of approximate repetitions in polyphonic MIDI files based on perceptive criteria. International Symposium on Computer Music Modeling and Retrieval (CMMR 2003), May 2003, Montpellier, France. pp.124-142, 10.1007/978-3-540-399001_13. hal-01161126

\author{
HAL Id: hal-01161126 \\ https://hal.science/hal-01161126
}

Submitted on 8 Jun 2015

HAL is a multi-disciplinary open access archive for the deposit and dissemination of scientific research documents, whether they are published or not. The documents may come from teaching and research institutions in France or abroad, or from public or private research centers.
L'archive ouverte pluridisciplinaire HAL, est destinée au dépôt et à la diffusion de documents scientifiques de niveau recherche, publiés ou non, émanant des établissements d'enseignement et de recherche français ou étrangers, des laboratoires publics ou privés. 


\title{
Automatic Extraction of Approximate Repetitions in Polyphonic Midi Files Based on Perceptive Criteria
}

\author{
Benoit Meudic ${ }^{1}$, Emmanuel St-James ${ }^{2}$ \\ ${ }^{1}$ Musical representation team - IRCAM \\ 1, place Igor-Stravinsky \\ 75004 Paris, France \\ meudicdircam.fr \\ ${ }^{2}$ LIP6/SRC, Université Pierre et Marie Curie \\ 4, place Jussieu \\ 75255 Paris, France \\ Emmanuel.Saint-James@lip6.fr
}

\begin{abstract}
In the context of musical analysis, we propose an algorithm that automatically induces patterns from polyphonies. We define patterns as "perceptible repetitions in a musical piece". The algorithm that measures the repetitions relies on some general perceptive notions: it is non-linear, non-symetric and non-transitive. The model can analyse any music of any genre that contains a beat. The analysis is performed into three stages. First, we quantize a MIDI sequence and we segment the music in "beat segments". Then, we compute a similarity matrix from the segmented sequence. The measure of similarity relies on features such as rhythm, contour and pitch intervals. Last, a bottom-up approach is proposed for extracting patterns from the similarity matrix. The algorithm was tested on several pieces of music, and some examples will be presented in this paper.
\end{abstract}

\section{Introduction}

Automatic music analysis is an increasingly active research area. Among the main tackled subjects, the search for musical patterns is at a central place. Indeed, most of the musical pieces are structured in various components ("phrases", "motives", "themes", "generative cells"...) that can naturally be associated with the notion of pattern. Considering the only notion of "pattern" simplifies (or postpones) the issue of making the distinction between the different natures of the components (theme, motive etc...) of a musical piece. However, answering to the question "what is a pattern?" is still rather difficult. Often, the notion of pattern can be linked with the notion of repetition: patterns emerge from repetition. But a pattern could also be defined by its salient boundaries, and then patterns would emerge from discontinuities in the music. Last, patterns can also be characterized as independent and coherent entities. Providing a definition is all the more difficult as we place ourselves in a musical 
context. Most of the time, patterns are linked with perceptive notions, which raises one question: can we consider as "patterns" the structural (one would say mathematical) regularities of a musical sequence, even if theses regularities are not perceived? Inversely, do perceived repetitions only correspond to exact repetitions? We think that the notion of similarity between two sequences plays an important role in the perception of patterns and it should be part of a pattern extraction system.

In this article, we have chosen to focus on perceptible musical structures. Moreover, we assume that musical structures can be induced from the extraction of repeated sequences (that we call patterns) and thus we address the issue of extracting "perceptible repetitions" from a musical piece.

\section{Background}

The literature is quite poor in algorithms that automatically extract patterns (perceptible repetitions) from polyphonic music.

An interesting method, starting from the audio, is proposed by Peeters [1]. It considers the signal as a succession of "states" (at various scales) corresponding to the structure (at various scales) of a piece of music. A similarity matrix is computed from feature vectors. The similarity matrix reveals large and fast variations in the signal that are analysed as boundaries of potential patterns. The patterns are then grouped according to their similarity. The method is relevant for pieces that contain salient transitions (timbre changes, important energy variations etc...), but could reveal less relevant for the detection of phrases in piano music.

Another method in Lartillot [2] starts from MIDI files and induces patterns by analysing the musical sequence in a chronological order. All the possible combinations of successive events contained in a temporal window of limited size are potential candidates for being a pattern. The notions of temporal context and expectation are modelized. However, if very promising, this method cannot analyse long sequences with too many events because it would require a too high computation cost. Moreover, polyphonic context (see 4.1 for definition) is not considered.

Rolland[3] proposes a model that uses dynamic programming. All pairs of possible patterns of a given sequence are compared. Pairs whose similarity value overlaps a given threshold are memorized into a similarity graph. An interesting optimisation of the computation time is proposed. However, the similarity measure that is used is linear which might not be cognitively relevant for us as we consider a pattern as a whole entity (see equation 1 in section 5.2.1). Moreover, the algorithm cannot extract patterns from unvoiced polyphonic music, such as piano music.

Meredith et all [4] propose a multidimensionnal representation for polyphonic music. The algorithm searches for different matchings of the geometrical figures of feature vectors by translating them in a multidimensionnal space. This approach is interesting because it offers a graphical answer to a musical issue. However, only exact repetitions can currently be extracted. The way to solve this issue seems to find relevant representations that could be identical for musical patterns that are perceptively 
similar but physically different. Otherwise, the repetition detection algorithm should be modified.

Last, Cambouropoulos [5] proposes to segment a MIDI file and then to categorize the segments in clusters. The segmentation algorithm uses a boundary detection function that computes the similarity between all the possible patterns of the sequence. Then, the segments are clustered according to a threshold that depends on the different distances between the segments. Once the clusters are computed, the distances between segments are computed again in order to optimize the current clustering, and the clustering function is called again until the clusters are found optimal. This method is interesting because it analyses whole sequences of polyphonic MIDI music, and the notion of context is originally used in one of the two steps of the analysing (the clustering). However, the segmentation step appears hazardous, and would require a high computational cost.

\section{$3 \quad$ Aims}

The algorithm we present in this article is a new model for extracting patterns from polyphonic MIDI sequences. Our aim is that the patterns we extract can be considered as components of the musical structure. In order to build a system the more general as possible, we do not consider any knowledge based on tonality neither we consider particular styles. Several perceptive notions are taken into account in the algorithm. However, in a first step, we have chosen to challenge the limits of a system that does not modelize temporal context or expectation. Indeed, the integration in the system of all the cognitive mechanisms that play a role in our perception of music is far too complex. Thus, we have to draw the limits of the model. The consideration of temporal context and expectation should be possible in a second step, but we first prefer to explore the limited system, and we will rely on the results to show that our method offers promising applications. Besides, we will not try to extract all but a set of significant patterns from polyphonic music. If our aim is that the extracted patterns are the most relevant of all the sequence, we consider as more important the relevance of the pattern itself.

\section{Introduction to the pattern extraction model}

\subsection{General considerations on patterns}

We have defined patterns as "perceptible repetitions in a musical sequence". This notion has to be refined before we describe in details the model.

An issue arises when refining the term 'repetition' of the definition. How to define the similarity between two sequences? An attempt to answer to this question is proposed in 5.2. 


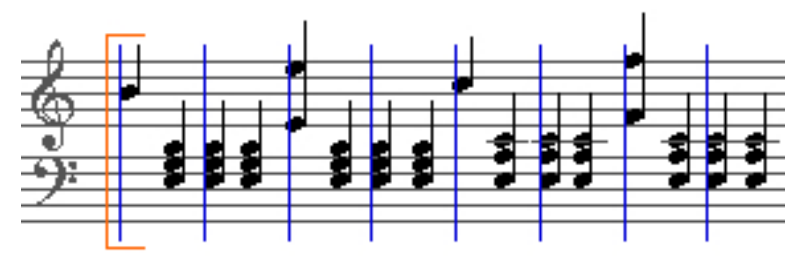

Fig. 1. Beginning of the Sonata for piano No9 EM from Beethoven. The first note and the three chords form a pattern. Transforming the three chords into three independent voices would not be perceptively relevant.

Another issue stems from the polyphonic context: is a pattern a single melodic line inside a polyphony, or is it itself a polyphonic component of a polyphony? For instance, let's consider the following extract of the 9nth Beethoven's sonata (Fig. 1). The sequence that is composed by the first four events can be called a pattern as it is repeated several times. This sequence is polyphonic (one note followed by three chords). Should we consider that the sequence of chords is composed by three different monophonic patterns that are superposed, or is it only one polyphonic pattern? Would it be relevant to dissociate the chords in as many components as there are notes inside, or should we consider them as entities? This also asks the question of the independence of a melodic line from its polyphonic context. Can we always isolate one or several monophonic lines, or do the superposition of several notes form a unique entity that cannot be decomposed? In several musical examples, there would be no reason to extract a single monodic line (see also Fig. 2).

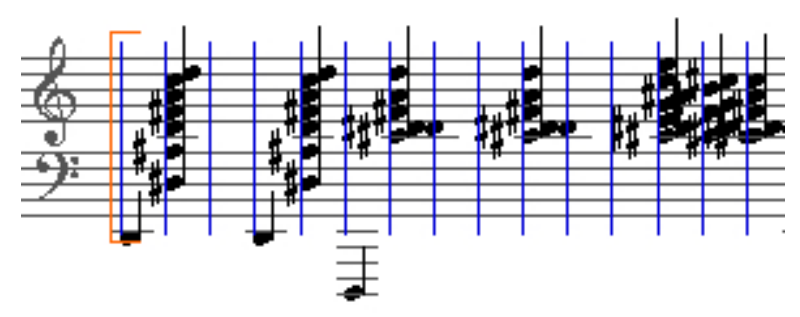

Fig. 2. A pattern extracted from the Sacral dance of the Rite of the spring from Stravinsky. A monodic voice could hardly be isolated from the polyphonic pattern.

We also claim that considering a single melodic voice without its polyphonic context is often nonsense, as the polyphonic context often plays an important role in the perception of the structure of the piece. For instance, if we consider the beginning of the Sonata AM d664 op121, 1rst Part from Schubert (Fig. 3), one can extract the melody from the voice at the top. However, the segmentation of the melody in different patterns (that correspond in musical analysis to the usual "antecedent-following" repeated one time with variation) is not trivial without information on the polyphonic context. Similarly, if we consider the beginning of the Intermezzo op117 no1 from 
Brahms (Fig. 4), we could extract the melody by hand with difficulties (it is not at the top of the voices, and it is repeated but with big variations), but it would not characterize the excerpt. Indeed, one could imagine the same simple melody in very different polyphonic contexts, divided in several different voices.

However, and this raises one of the main issues, the only melodic line should also be taken into account, for instance when it follows the model 'melody + accompaniment'. Indeed, in this case the melodic line could be separated from the accompaniment and considered as a monophonic pattern that would be repeated in the following music sequence, but with different accompaniment, or with melodic variations. This is mainly a perceptive issue that is difficult to solve with only one rule.
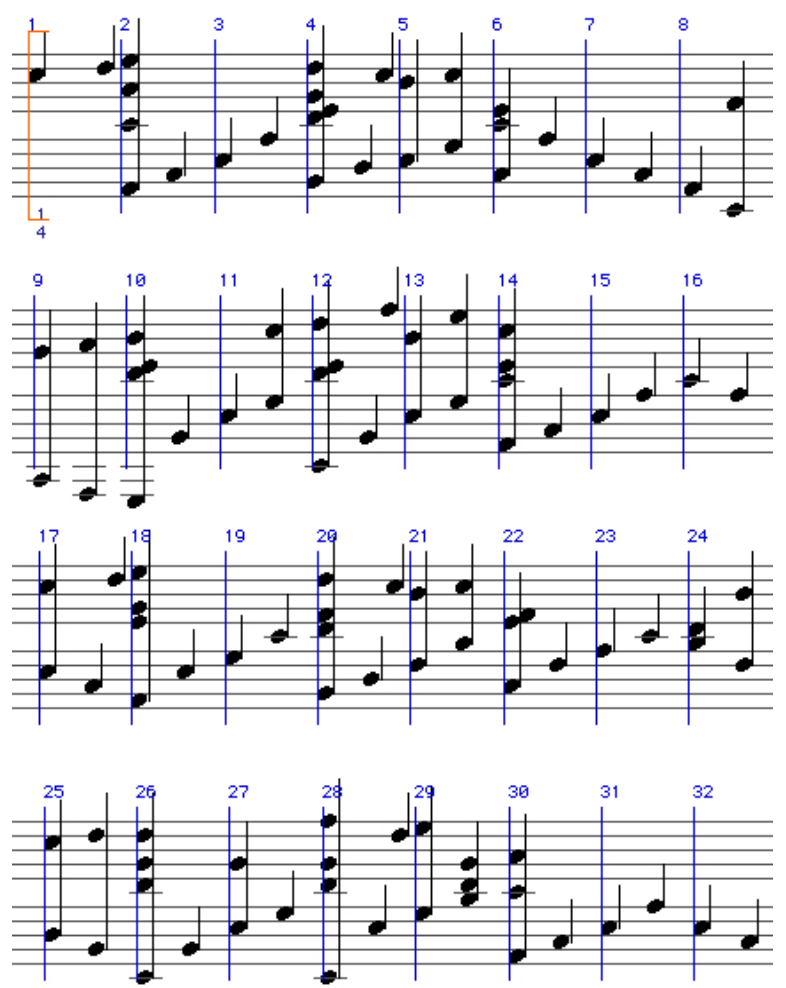

Fig. 3. Beginning of the Sonata AM d664 op121, 1rst Part from Schubert. The four patterns correspond to the structure antecedent1-following1-antecedent2-following2. This structure could not be extracted from the consideration of the only melodic line.

Sometimes, the solution is trivial. For instance, melodic lines are often difficult to follow in Bach's Fugues, but fortunately, they appear at the beginning of the fugue one after the other, which let us time to memorize them. In this case, the pattern first appears in a monophonic context (the Fugue's subject) and then is repeated and varied in a polyphonic context. The issue is not to extract a pattern (monophonic or polyphonic) from polyphony but to recognize a known (or memorized) monophonic pat- 
tern inside polyphony. However, in some pieces such as canons, only the beginning of the pattern appears in a monophonic context. The repetition of the pattern covers a part of it. Thus, in a polyphonic context, music cannot always be segmented in individual successive segments (that is often proposed in several musical theories) and possible coverings between the structural components must be taken into account.

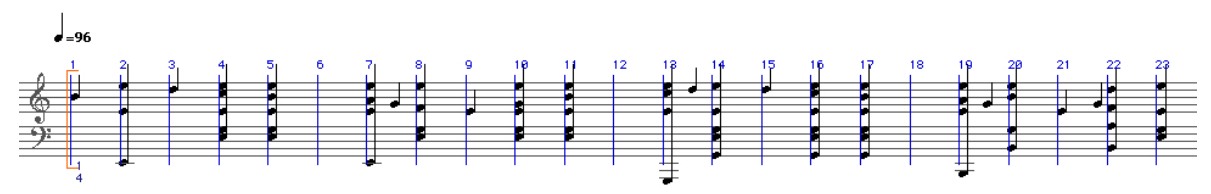

Fig. 4. Beginning of the Intermezzo op117 no1 EM from Brahms. The sequence can be divided in four similar patterns (1-6, 7-12, 13-18 and 19-24) Polyphonic context is part of the structure of the sequence. The only melody line with another polyphonic context would not be stated as similar to this one.

Whatever the situation would be, we believe that the polyphonic context plays an important role. Even if a single melodic line could be extracted from the polyphony, the polyphony should be associated to the melody in most of the cases. One could say that when remembering a polyphonic excerpt, we often sing a single melodic line, but this is due to our physical impossibility to sing a polyphonic sequence, and often when singing the melodic line, we hear (but don't sing) the polyphonic context (at least the harmony) that was associated with it. It means that we have memorized it, and that we take it into account in our comparisons with other sequences. In this article, we propose to define a pattern as a polyphonic component of a polyphonic sequence.

\subsection{General architecture of the model}

Our model analyses a MIDI file that contains the onset (in milliseconds), the pitch (in midicents) and the duration features. The dynamic and the channel features are not considered.

The model is composed of two main algorithms:

- the first algorithm computes several similarity matrices from a quantized MIDI file (Section5).

- the second algorithm extracts patterns from the similarity matrices (Section 6).

In the first algorithm, we first quantize and segment the MIDI sequence with an algorithm proposed in Meudic [6]. The boundaries of the segments correspond to the downbeats of the music. The initial sequence of MIDI events is thus considered as a sequence of beat-segments. Each beat-segment (b.s) is itself a sequence of MIDI events (see Fig. 5). Then, given a length L (in number of beats), each sequence of b.s of length $\mathrm{L}$ is compared with all the other sequences of same length. For each comparison, three similarity values are computed (corresponding to three different features: pitch intervals, pitch contour and rhythm) and associated with the events of the two 
sequences that have been found similar (we call them "templates"). All the similarity measures are then stored in similarity matrices. The measures of similarity we use are presented in 5.2.

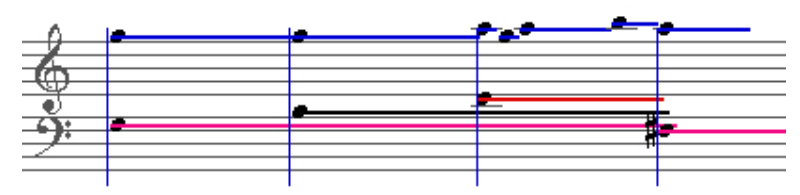

Fig. 5. A sequence of beat-segments (b.s) extracted from the "Variations Goldberg" from Bach. The vertical lines delimit the beat segments. Horizontal lines are the durations of each event.

In the second algorithm (section 6), patterns are extracted from the similarity matrices. First, the matrix cells (a matrix cell is a couple of two sequences of b.s of same length) are filtered and clustered according to their similarity value. Then, patterns are extracted in a bottom-up approach that starts from the different clusters of cells and then groups them in new (and often smaller) clusters with longer cells.

\section{The similarity matrices}

\subsection{The computation of a matrix}

A similarity matrix (Fig. 6) stores the similarity values between the pairs of b.s (beat.segment) sequences of same length $\mathrm{L}$ (in number of beats). The units of the vertical and horizontal axis are expressed in number of beat-segments. We consider the matrix as symmetric (see discussion in 5.2.5). Each cell refers to a pair of b.s sequences. The vertical and horizontal positions of each cell are the two beginning positions (in number of beats) of the two corresponding b.s sequences.

Each similarity measure provides a real value between 0 and 1 that states how similar are the compared sequences ( 1 is for identical).

In the model, sequences of b.s are of same length (length is expressed in number of b.s) so that each position of b.s in a given sequence can be matched with the same position of b.s in the other sequence.

The choice of the length L can appear somewhat arbitrary. The issue is to find the "right level" between the maximum length of patterns that cannot be divided in several parts and the minimum length of patterns that can be divided. Indeed, we think that some patterns must be considered as whole entities (see sub-section 5.2.2) and not as the concatenation of smaller patterns. Two such patterns (sequences of beat-segments) can be similar while their components (the beat-segments), if considered individually, 
are different. We think that the maximal length of such patterns could be linked to the limits of our short-term memory: when listening to a musical sequence, we compare what we are hearing with what we have already listened. If what we are hearing is a variation of something already listened, we will match the two sequences. We match the sequences because they share common aspects. The question is: what is the minimal number of common aspects, and the maximal distance between the aspects, that is required to initiate a match between the sequences. In other words, what is the maximal size of a pattern (for us, the size is in number of beat-segments) that must be considered for matching it with another pattern?

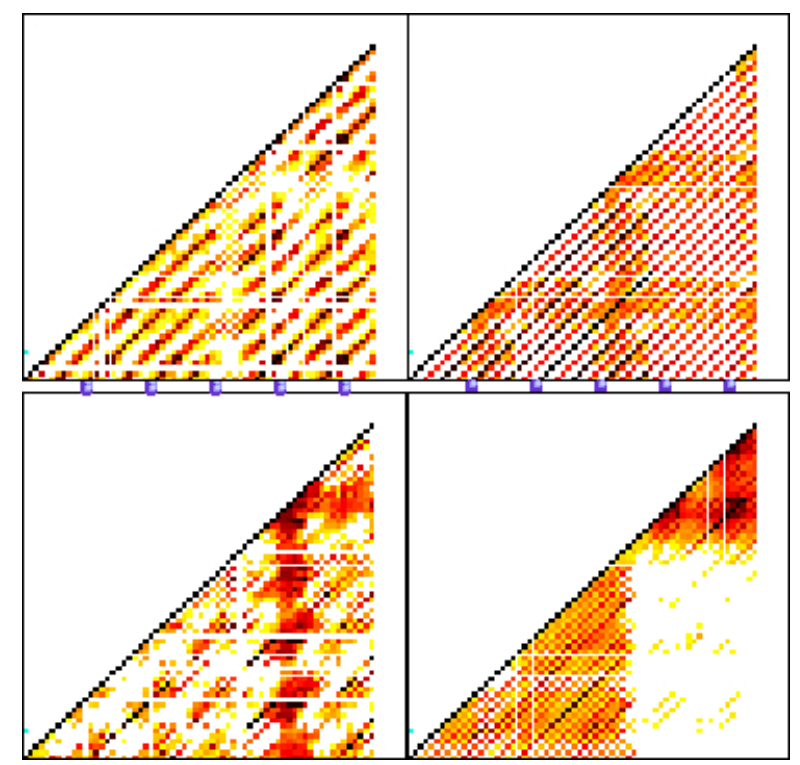

Fig. 6. Four different similarity matrices from first 30 seconds of (from left to right and top to bottom): Sonata AM d664 op121 $2^{\text {nd }}$ Part from Schubert, 1rst Gymnopedie from Satie, 1rst and $3^{\text {rd }}$ part of the Sacral dance of the Rite of the Spring from Stravinsky. White areas correspond to dissimilar cells. The unit for both vertical and horizontal axis is expressed in number of beat-segments (one cell per beat-segment).

In our tests, we have decided to define a "blurred" length of pattern L: we compute the similarity between pairs of sequences of length L, L-1, L-2 and L-3, and we choose the best (the higher) similarity value (a decreasing coefficient is applied to the similarity value when the length of the sequence decreases, in order to support longer sequences).

\subsection{The similarity measures}

In this part, we define several similarity measures between two b.s sequences of given length. 
We compute three different similarity values by considering three different sets of features: pitches (chords, pitch intervals etc...), pitch contours (contour at the top and at the bottom of the polyphony) and rhythm. The similarity values are computed in respect with some cognitive aspects (see sub-section 5.2.1). Each time a similarity value is computed between two sequences seq 1 and seq 2 , it is associated with two "templates", that is to say the events of seq1 similar to seq2 (template 1 ) and the events of seq 2 similar to seq1 (template2). These templates will be used to refine the kind of similarity relation that exists between the two sequences (see sub-section 6.1).

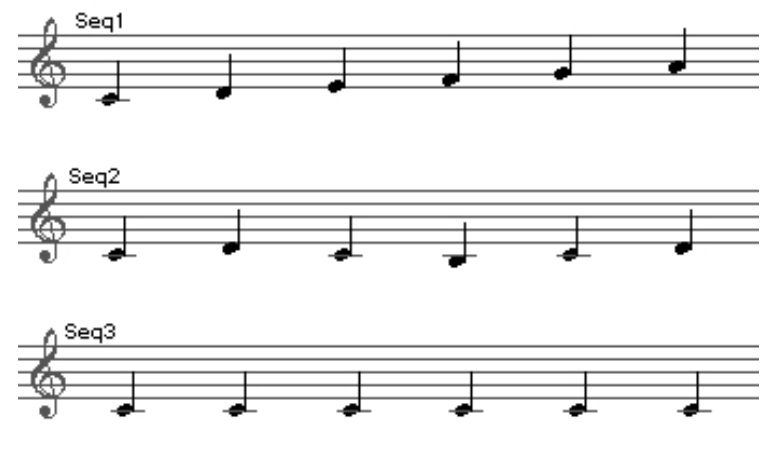

Fig. 7. Seq 1 and Seq 3 have similar consecutive intervals but are globally different. Seq2 and Seq 3 have also similar consecutive intervals but are globally more similar. Intervals between not consecutive events have to be considered to measure this difference.

\subsubsection{Similarity in a polyphonic context}

We assume that the notion of similarity between two polyphonic musical sequences makes sense. No information is available on the different voices of each sequence. Computing the intersection between the two sequences would appear as an intuitive way to measure what is common between the two sequences. However, the intersection could be empty while the two sequences would be perceived as similar.

Thus, we state that a sequence $\mathrm{x}$ is similar to a sequence $\mathrm{x}^{\prime}$ if $\mathrm{x}$ is approximatively included in $x^{\prime}$. For instance, when listening to music, we try to associate one sequence already heard with the current sequence we are hearing. We do not intersect the two sequences, but we evaluate the similarity between one sequence and a reference one. Thus, in our model, we understand similarity between two sequences $\mathrm{x}$ and $\mathrm{x}^{\prime}$ as the distance from $x$ to a certain sequence $\operatorname{sub}\left(\mathrm{x}^{\prime}\right)$ included in $\mathrm{x}^{\prime}$.

\subsubsection{Cognitive aspects of the similarity measure}

First, a musical sequence of b.s is considered as a whole entity (it may contain an abstract cognitive structure) and not solely as the concatenation of smaller entities. Thus, the similarity measure is not linear (see equation 1). Indeed, we think that it is necessary to consider the relations between non-adjacent events. Theses relations play a role in the cognitive process for recognizing the similarity between two sequences. 
For instance, two sequences that are locally similar but that have different global pitch range may not be very similar (see Fig. 7).

$$
S\left(x, x^{\prime}\right)+S\left(y, y^{\prime}\right) \neq S\left(x y, x^{\prime} y^{\prime}\right) \text {. }
$$

$\left(\mathrm{S}\left(\mathrm{x}, \mathrm{x}^{\prime}\right)\right.$ designs the similarity value between sequences $\mathrm{x}$ and $\mathrm{x}^{\prime}$, and $\mathrm{xy}$ designs the concatenation of sequence $x$ and $y$ )

Another cognitive aspect is that our similarity measure is not symmetric in a polyphonic context (equaltion 2).

$$
\mathrm{S}\left(\mathrm{x}, \mathrm{x}^{\prime}\right) \neq \mathrm{S}\left(\mathrm{x}^{\prime}, \mathrm{x}\right) \text {. }
$$

In our model, a sequence $\mathrm{x}$ is similar to a sequence $\mathrm{y}$ if $\mathrm{x}$ is approximatively included in $y$. However, this do not mean that $y$ is included in $x$ and thus we do not know if $\mathrm{y}$ is similar to $\mathrm{x}$. Moreover, if the first half of $\mathrm{x}$ is included in $\mathrm{y}$, and the second half of $\mathrm{y}$ is included in $\mathrm{x}$, the similarity cannot be determined. To compute the similarity, we must determine either if $\mathrm{x}$ is entirely included in $\mathrm{y}$ or if $\mathrm{y}$ is entirely included in x (see Fig. 8).

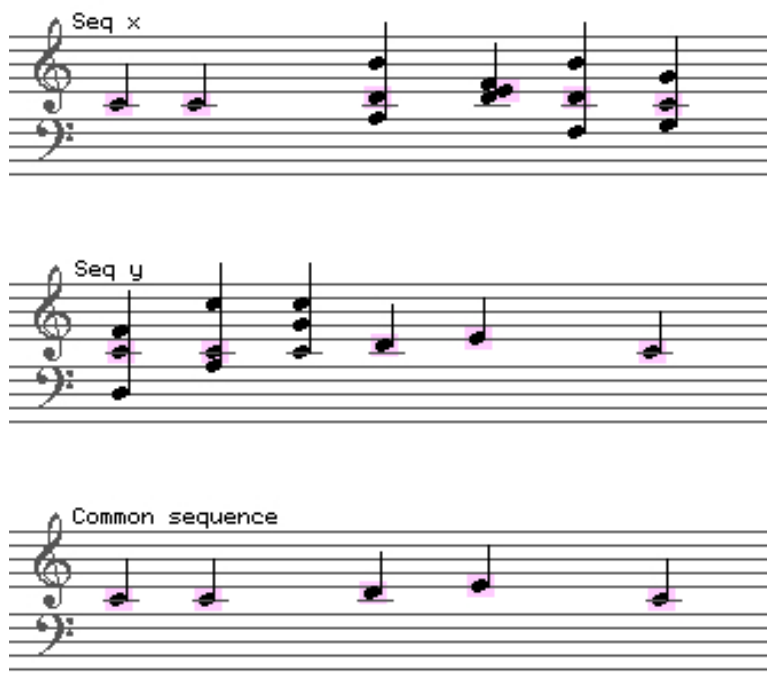

Fig. 8. Sequence $x$ and sequence y have common events but are not very similar because none of the sequences is included into the other.

Last, according to Tversky [7], the similarity measure is not transitive, that is to say the triangular inequality is not true (equaltion 3 ).

$$
\mathrm{S}(\mathrm{x}, \mathrm{y})+\mathrm{S}(\mathrm{y}, \mathrm{z}) \leq \text { or } \geq \mathrm{S}(\mathrm{x}, \mathrm{z}) \text {. }
$$

For instance, $\mathrm{z}$ can be a variation of $\mathrm{y}$ that is a variation of $\mathrm{x}$. But $\mathrm{z}$ can be very different from $\mathrm{x}$ and thus not judged as a variation of $\mathrm{x}$.

The three different cognitive aspects have been considered in our model of similarity. We will now provide more details on the different similarity measures. 


\subsubsection{Similarity measure for pitches}

In this section, we consider the chords and the pitch intervals features. A similarity value is computed from two b.s (beat.segments) sequences seq1 and seq 2 of same length.

The only events falling on the downbeats are considered. This may be arguable, but two reasons have conducted this choice:

- considering all the polyphonic events would require too much running time

- the downbeats are often perceived as salient temporal position. Two sequences whose pitches coincide on the downbeat but differ elsewhere are often recognised as very similar (this has been confirmed in our experiments).

Usually, a downbeat event (dwb.event) is a chord, but it can also be a note or a rest.
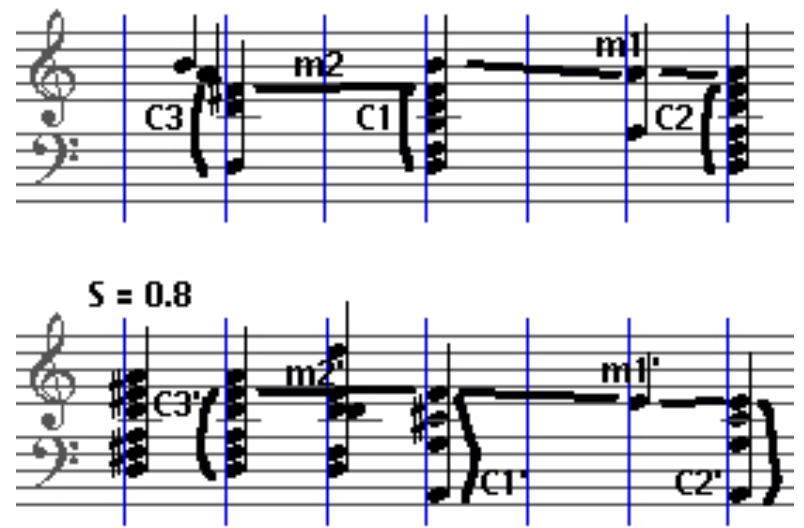

Fig. 9. Two similar patterns from Sonata DM d664 op121 $2^{\text {nd }}$ Part from Schubert. A reference pattern (the above one) is compared to another pattern (the similarity measure for pitches is 0.8 on 1). Durations are not represented. The high similarity can partially be explained by the fact that: some chords are repeated $(\mathrm{C} 1$ is similar to $\mathrm{C} 2$, and $\mathrm{C} 1$ ' ' is similar to $\mathrm{C} 2$ '), $\mathrm{C} 3$ is similar to $\mathrm{C} 3$ ', the melodic line $\mathrm{m} 1$ is similar to $\mathrm{m} 1$ ', and the melodic line $\mathrm{m} 2$ is similar to $\mathrm{m} 2$ '.

We focus on two musical aspects: the vertical and the horizontal one.

1. The vertical aspect concerns the repetition of chords. A chord is represented by the intervals between the lower pitch and the other pitches of the chord. The intervals are computed modulo 12 .

2. The horizontal aspect concerns the similarity between melodies. A melody is any sequence of consecutive pitches.

\subsubsection{The repetition of chords}

In order to consider all the possible relations between non-adjacent chords, the global similarity results from the mean of the similarity values between all the pairs of chords in seq1 and their corresponding pairs in seq2 (see Fig. 9). 
The similarity function between a pair P1(C.1, C.1') and a pair P2(C.2, C.2') is provided in the following listing.

Listing in Lisp of the similarity function between two pairs of chords C.1, C.1' and C.2, C.2':

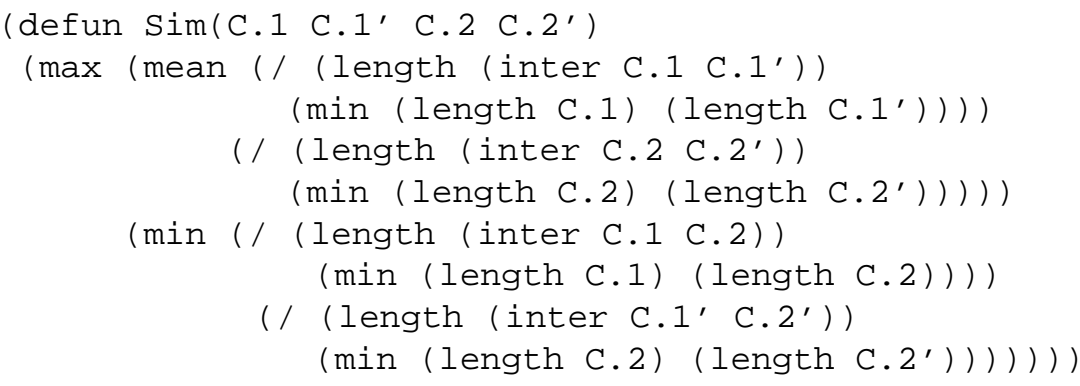

First, the function computes two values (see fig. 9): the percentage of the common notes between the chords in the pair $\left(\mathrm{C} 1, \mathrm{C} 1^{\prime}\right)$ and the chords in the pair $\left(\mathrm{C} 2, \mathrm{C} 2{ }^{\prime}\right)$. If $\mathrm{C} 1$ and $\mathrm{C} 1$ ' have common notes and $\mathrm{C} 2$ and $\mathrm{C} 2$ ' have not, the pairs $\mathrm{P} 1$ and $\mathrm{P} 2$ are still perceived as similar. Thus we consider the mean of the two values.

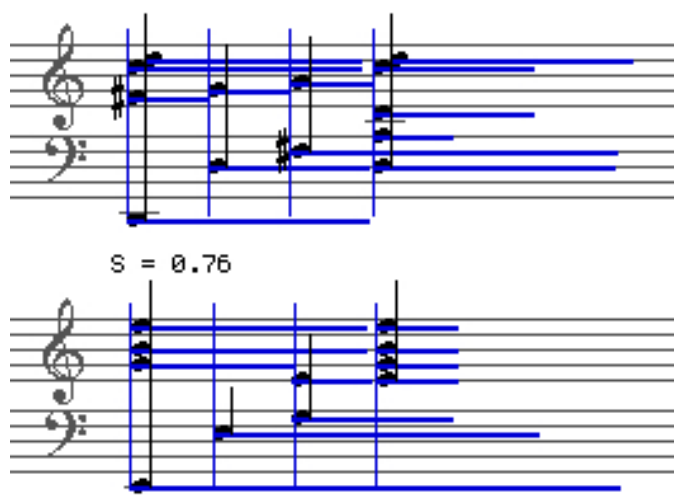

Fig. 10. Two similar patterns from intermezzo op $119 \mathrm{n}^{\circ} 3$ from Brahms. The contours at the top are similar (contour D-D and contour E-E). Pitches in the middle of the two sequences are not in the contours because the durations (horizontal lines) of the first pitches $\mathrm{D}$ and $\mathrm{E}$ of each contour cover them.

Then, the function computes another two values: the percentage of the common notes between the chords in the pair $(\mathrm{C} 1, \mathrm{C} 2)$ and the chords in the pair (C1', C2'). If $\mathrm{C} 1$ and $\mathrm{C} 2$ have common notes and $\mathrm{C} 1$ ' and $\mathrm{C} 2$ ' have not, the two pairs can still be very different. Thus, we consider the min of the two values instead of the mean.

\subsubsection{The similarity between melodies}


Considering two sequences of beat-segments seq 1 and seq2, a function computes the best fitting between seq1 and different transpositions of seq2. The aim is to find melodic lines in seq2 that are included in seq1 (see Fig. 9). To simplify the description of the model, we consider that each down-beat of each beat-segment correspond to one chord. Thus, we compare two sequences of chords seq1 and seq2. Seq1 and seq2 have same number of chords L.

The model contains L-1 iterations. Each iteration i compares sequences seq1 and seq 2 whose first i-1 chords have been deleted. The same comparison function is processed for each iteration. This function outputs a similarity value between 0 and 1 . The global similarity value between the two sequences seq 1 and seq 2 is the mean of L-1 similarity values that are computed for each iteration.

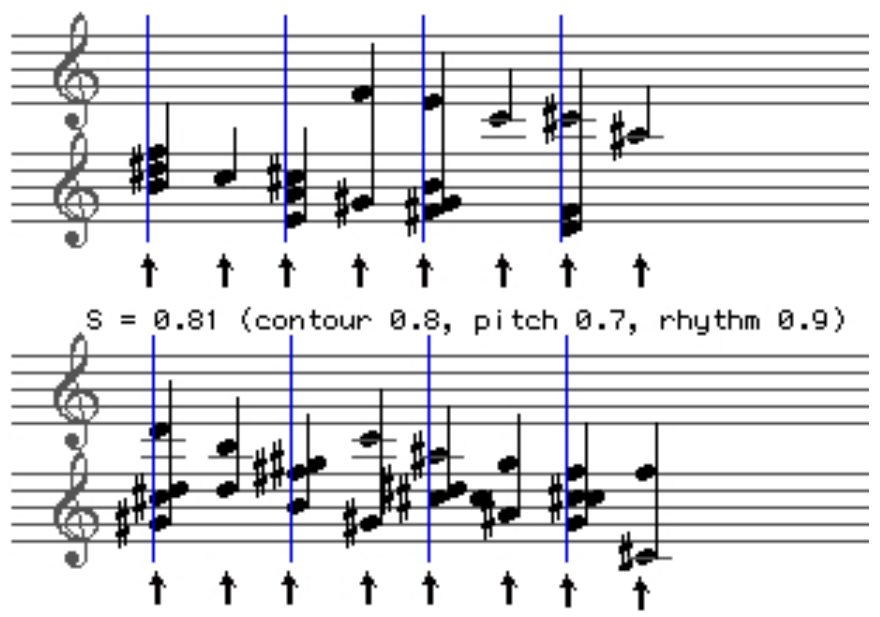

Fig. 11. Two similar patterns from Pierrot Lunaire from Schoenberg. Rhythm is very similar. Corresponding onsets are marked with an arrow.

We will now describe the comparison function for the first iteration. Considering one pitch of the first chord of seq1, pitch(i)(chord1(seq1)), we try as many transpositions of seq 2 as there are pitches on the first chord of seq2. A transposition consists in transposing the pitches of seq2 so that one pitch of (chord1(seq2)) is equal to the pitch pitch(i)(chord1(seq1)). The length (in number of chords) of the longest intersection of seq 1 with the different transpositions of seq 2 is memorized. This length is divided by the total number of chords $\mathrm{L}$ (remind that seq 1 and seq 2 have same length $\mathrm{L}$ ). The iteration of the process to the $\mathrm{N}$ pitches of the first chord of seq 1 provides $\mathrm{N}$ values between 0 and 1 . The final result of the comparison function is the mean of the $\mathrm{N}$ values. Then, the comparison function is applied to the second chord of seq1 and seq2. 


\subsubsection{Similarity measure for contours}

Our model compares the upper and lower contours of two b.s sequences seq1 and seq2 of same length (see Fig. 10).

As above, the only events falling on a downbeat (dwb.events) are considered. An up (down) contour is the sequence of the upper (lower) pitches of the dwb.events. If the duration of a pitch $\mathrm{p} 1$ covers the duration of the following pitch $\mathrm{p} 2$ and if $\mathrm{p} 1$ is higher (lower) than $\mathrm{p} 2$, then $\mathrm{p} 2$ will not be part of the up (down) contour.
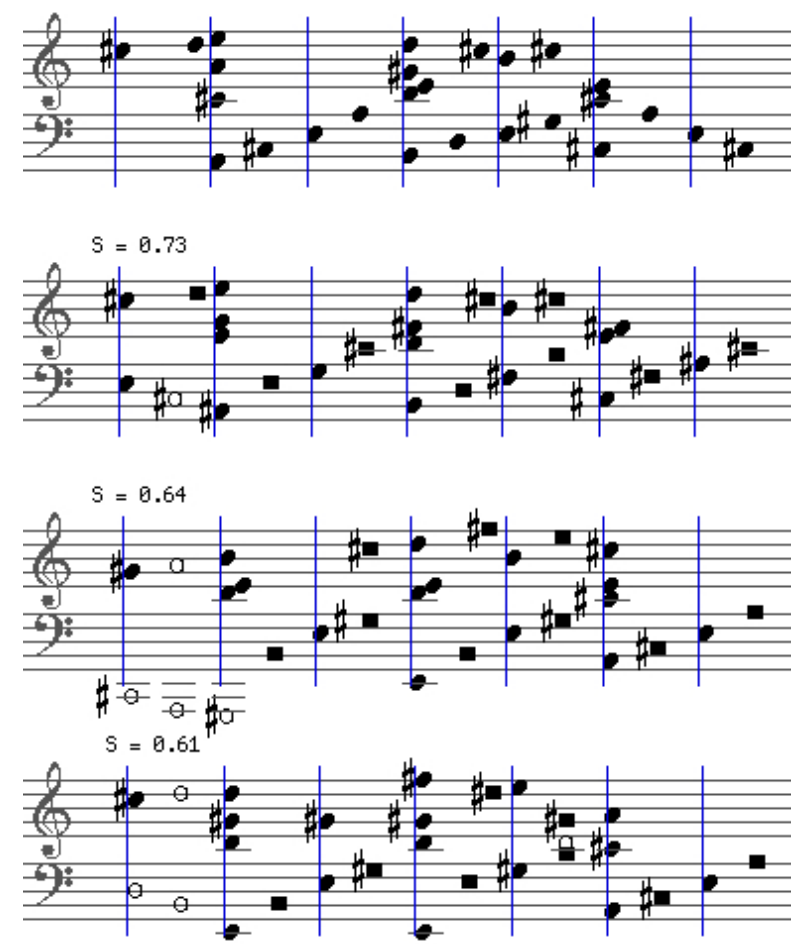

Fig. 12. Three patterns similar to the above pattern in Sonata AM d664 op121, 1rst Part from Schubert. The similar patterns are sorted according to their decreasing global similarity value $S$. The square symbols only determined the similarity for rhythm. The oval symbols determined similarity for both pitches and contour.

Each contour of each sequence is compared with the two other contours (up and down) of the other sequence. The similarity value between two contours is the mean of the similarity values between all possible pairs of intervals between corresponding pitches of the two contours. The numerical values we use for comparing two intervals int 1 and int 2 are the following:

- 0.7 for down contours if int $1=$ int 2

- 1 for other contours if int $1=$ int2

- 1 for up contour if sign(int1) = sign(int2) and abs(int $1-$ int2) $<4$

- 0.7 for other contours if $\operatorname{sign}($ int1 $)=\operatorname{sign}($ int2 $)$ and abs $($ int $1-$ int2 $)<4$ 
- $\quad 0.4$ if $\operatorname{sign}($ int 1$) * \operatorname{sign}($ int 2$)=0$ and if abs(int $1-$ int 2$)<3$

- 0 if sign(int 1$) * \operatorname{sign}($ int2 $)=0$ and if abs(int $1-$ int 2$)>=3$

- 0.1 if abs(int $1-$ int2) $>9$

- 0.3 if abs(int $1-$ int 2$)>3$

- 0 else

\subsection{6 similarity measure for rhythm}

Our model compares the rhythmic structure of two sequences of b.s seq1 and seq2 of same length (see Fig. 11). In a first step, seq1 and seq2 are normalized so that the total duration of the b.s will be the same for seq1 and seq2. Then, for each b.s, onsets (temporal positions) in seq1 are associated to the corresponding onsets in seq2. Two onsets of two b.s form a pair if they share similar temporal positions in the b.s. If an onset of one sequence does not form a pair with an onset of the other sequence, then it is deleted.

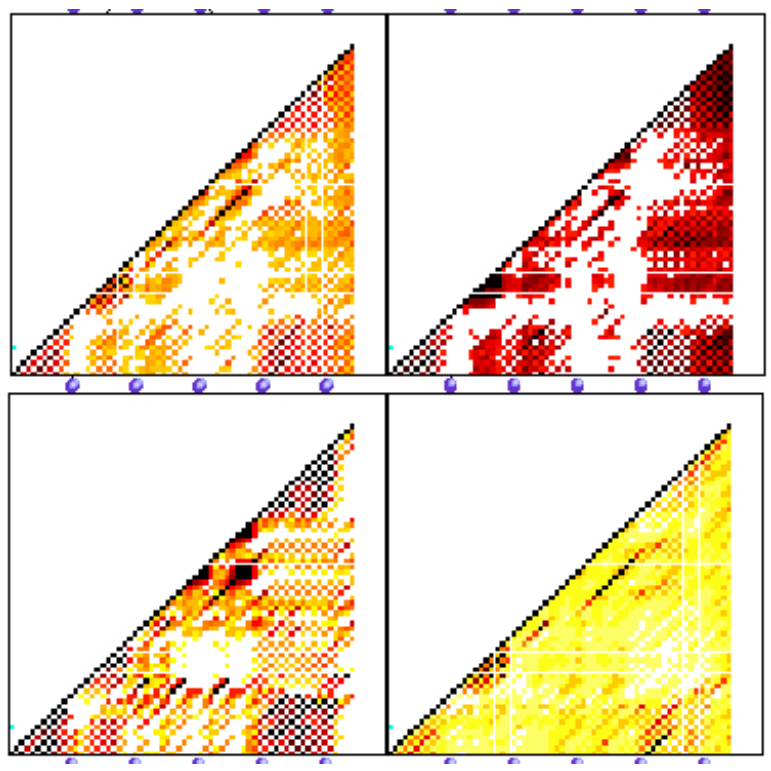

Fig. 13. Four similarity matrices from Sonata for piano $\mathrm{N}^{\circ} 9 \mathrm{EM}$ from Beethoven (from left to right and top to bottom): overall similarity measure, similarity for rhythm, similarity for contour and similarity for pitches. The three measures are very different (see Fig. 15 for comparison). The matrix corresponding to rhythm is less informative than the other matrices because dark diagonals do not clearly emerge.

The similarity between two sequences of b.s is the mean of the similarity between each corresponding b.s (as seq1 and seq 2 have same length, each b.s of seq1 correspond to one b.s of seq2). The similarity between two corresponding b.s is the mean of the the addition of the similarities between each pair of corresponding onsets divided by the total number of onsets of the reference b.s. The similarity between corresponding 
onsets is proportional the length of the intersection of the corresponding durations (an approximation value of the durations is considered for the intersection).

\subsubsection{Overall similarity measure}

Each of the three above measures (pitches, contour and rhythm) computes a similarity value between each pair of sequences of length $L$ contained in the musical sequence we analyse. The results can be represented in three different similarity-matrices that can be analysed separately. Sometimes, it appears that rhythm, pitches and contour play a different role in the similarity measure, and sometimes the similarity matrices for the different measures are very similar (see Fig. 13 and Fig. 15).

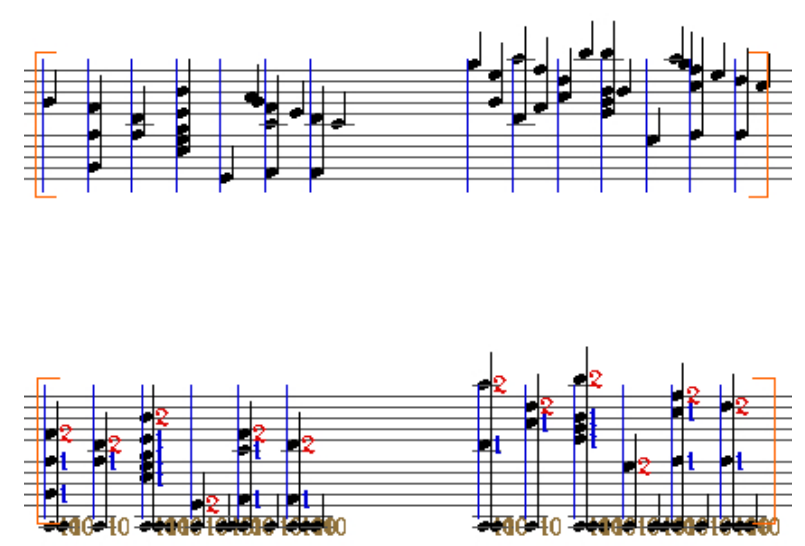

Fig. 14. Detail of one cell of the overall similarity matrix (Fig. 13). Above, the two compared sequences of the cell. Below, the similar events of each sequence (the templates). Numbers 1, 2 and 10 correspond to similar pitches, contour and rhythmical events. As rhythmical similarity do not considers pitches, it is represented by the events at the bottom of the sequence with static pitch $\mathrm{C}$.

The three measures can also be linearly combined into a global similarity measure (see Fig. 12 and Fig. 14 for similarity between sequences and Fig. 13 and Fig. 15 for similarity matrices). In this case, different weights can be applied to the different measures. An algorithm could be used to determine the best weightings by comparing the output of the similarity measures to a set of expected similarity-matrix. In our experiments, we have chosen to give the same weight to the different measures.

Due to the non-symmetrical relation (see equation 2), the similarity value between two non-ordered sequences seq 1 and seq 2 is composed of two different values: S(seq1, seq2) and $S$ (seq2, seq1). In our model, we have chosen not to consider the two different values and thus we only consider the greater value. Doing that, our similarity matrix is symmetric and we represent only half of it. 


\section{Pattern extraction from the similarity matrix}

Several different types of information can be extracted from the matrices: general evolution for rhythm, pitch intervals or pitch contours, local repetitions of cells, areas of structural changes, etc...

All of theses informations could be linked with the notion of pattern. However, in this part, we will only focus on the extraction of "the most important" patterns.

Defining such patterns is quite difficult, as there are no objective criteria to characterize them. One would agree to say that the "most important" patterns are the ones that are perceived as the "most salient" in the musical sequence: thus the attribute "most important" is related to perceptive criteria.

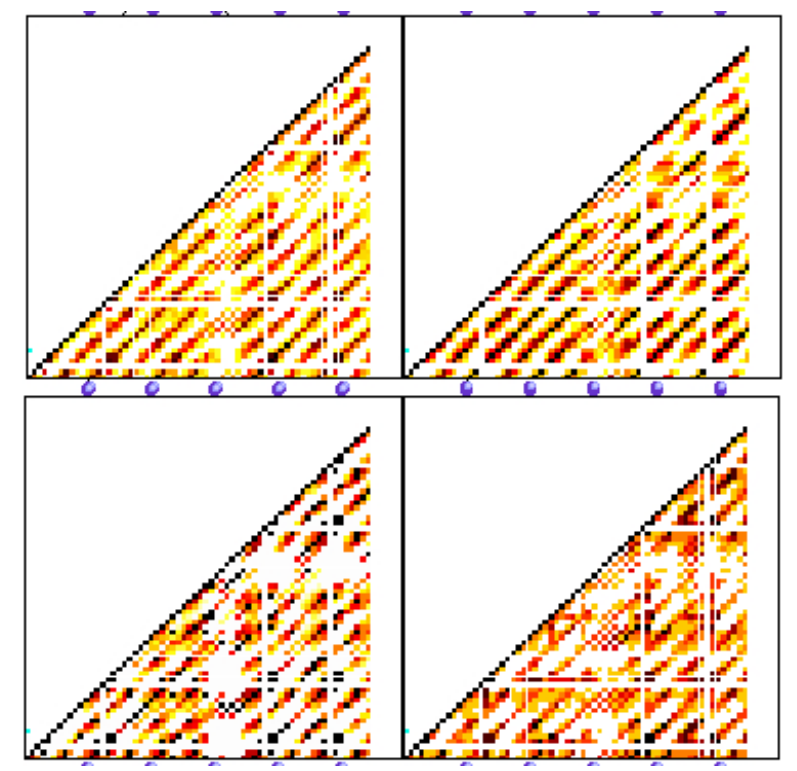

Fig. 15. Four similarity matrices from Sonata DM d664 op $1212^{\text {nd }}$ Part from Schubert (from left to right and top to bottom): overall similarity measure, similarity for rhythm, similarity for contour and similarity for pitches. The matrices of the three different measures are very similar (see Fig. 13 for comparison).

We think that the musical temporal context would play an important role in the definition of those criteria but once again, we will try not to consider it. Two other criteria are often found in the literature: the length of the pattern, and the number of its repetitions. However, it is quite difficult to combine the two criteria: the "most important" patterns often appear as a compromise between the two.

For instance, the same accompaniment can be found all along a musical piece. This pattern is very often repeated and thus very important in regards with the second criterion. However, it rarely appears to us as the "most important" pattern. Inversely, musical pieces that are exactly repeated two times can be seen as two very long patterns but they are not the most relevant for us because the repetition is trivial. 
We will now propose a method for extracting patterns that could be "the most important ones". First, we propose to filter and cluster the cells of the matrix. Then, we propose to concatenate the cells in long patterns.

\subsection{The filtering/clustering of the cells of the similarity matrices}

As we are looking for repetitions, we focus on the patterns that are very similar. For that, we choose a threshold that selects the only cells with the higher similarity values (see Fig. 16).

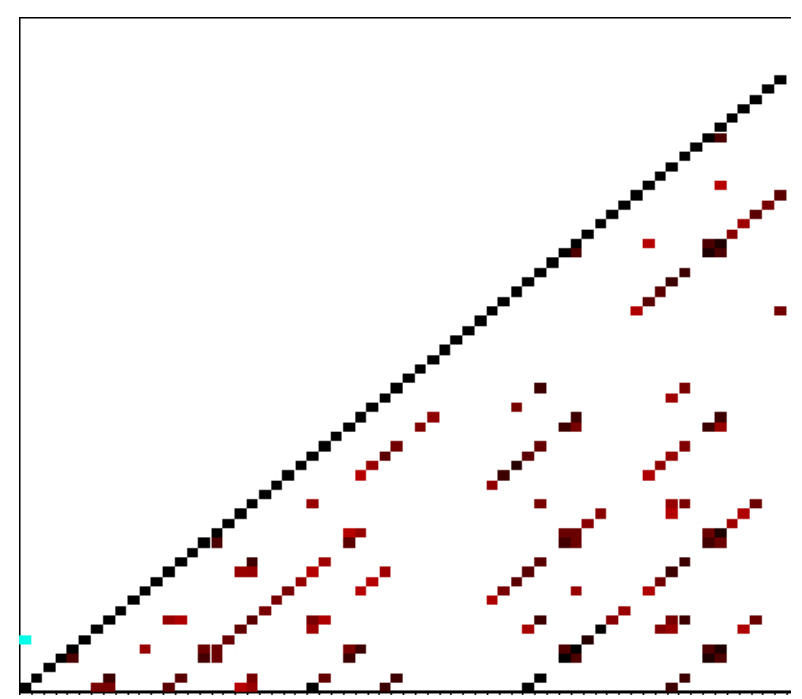

Fig. 16. A filtered similarity matrix from Sonata DM d664 op $1212^{\text {nd }}$ Part from Schubert (the initial matrix is in Fig. 15). The most similar patterns appear (diagonal lines).

Then, we cluster the cells: each horizontal line of the similarity matrix represents all the similarity values between a reference sequence s(ref) and all the sequences of the line s(i). The high similarity values reveal high similarity with the reference sequence, but they do not reveal the kind of similarity, which has to be evaluated. For that, we compare the templates of the sequences. The template of seq1 (respectively seq2) contains the events of seq1 (resp seq2) similar to seq2 (resp seq1) (see Fig. 14). Sequences that have very similar templates are considered as sequences that have same kind of similarity.

\subsection{The extraction of patterns}

We define a bottom-up approach for pattern extraction that starts from the clustered cells and builds new patterns by concatenation of the consecutive cells of a same diagonal line. 
The algorithm proceeds along the matrix from the lowest horizontal line to the upper one. For instance, in Fig. 17, the lowest horizontal line contains 8 cells. Only 6 of theses cells are clustered together. If we consider the second horizontal line, 6 cells can be concatenated to the 6 clustered cells of the first line. Thus, we have extracted 6 patterns of length " 2 cells". As there are no cells in the third line, we stop the process.

In a second step, we associate to each pattern the corresponding horizontal lines of the matrix (see 6.1). Theses lines contain clustered cells that could be concatenated and that could form new patterns (see Fig. 17). Theses patterns could then be added to the six other ones, depending on their kind of similarity with them. In Fig. 17, we highlight the 6 extracted patterns and a pattern that has been added in a second step (the last one on the right of the figure).

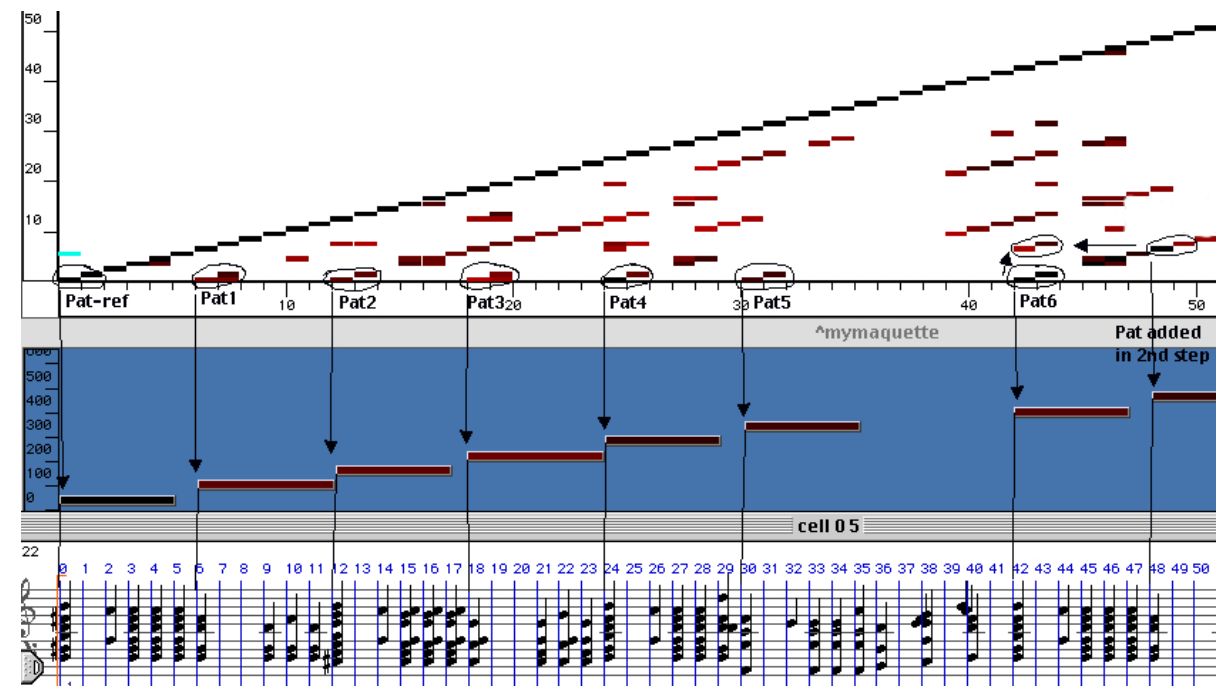

Fig. 17. (from top to bottom) The filtered similarity matrix from Sonata DM d664 op121 $2^{\text {nd }}$ Part from Schubert, the extracted patterns (each horizontal segment is an instance of the same pattern) and the corresponding musical sequence (the graduation of the matrix and the musical sequence is in "number of beat-segments").

We have tested several different musical pieces, and patterns that are part of the musical structure were often extracted. All of the musical examples that have been presented along the paper have been analysed, and the extracted patterns could always be associated with the musical structure of the piece. For other pieces, some patterns could be found that do not begin or end at the right temporal position in the sequence. Together with this issue, the consideration of all the notes of the polyphonic context was sometimes a constraint for recognizing the repetition of the only melodic line (for instance in canons pieces). The relations between the two (polyphony and the melodic line) is one of the main issue of pattern extraction. 


\section{Conclusion}

We have presented a general system for pattern extraction from polyphonic musical sequences. The notion of perceptible pattern has been discussed in the context of polyphonic music. A global similarity measure that takes into account rhythm, pitches and contour in a polyphonic context has been proposed. A method for extracting patterns from similarity matrices has been described and we have provided several musical examples for illustration. The method we propose could be used in several different musical applications (extraction of some components of musical structure, motive discovery, characterization of different musical pieces from their rhythmical or pitch components, search of allusions to a given pattern in a database of Midifiles). The similarity measure could also be adapted for applications such as "query by constraints": instead of humming a pattern, one could specify it with constraints on pitches or rhythm. The algorithm could then extract several patterns from different musical pieces with same rhythmical or pitch profile.

In future work, we plan to integrate temporal context in the model, in such a way that both polyphonic and temporal context are taken into account in the computation of the similarity matrices. We also plan to consider all the events of the sequence in the similarity measure and not only the events whose onsets correspond to the downbeat. Sequences with very different rhythm could then be compared and the quantifying step would not be necessary anymore. A prototype model has already been implemented. The only modification that we have made to the model concerns the algorithm that selects the sequences to compare. The similarity algorithm was not modified.

We think that our model considers interesting polyphonic aspects of music that are rarely taken into account, but several other issues remain to be solved, particularly the extraction of relevant patterns from the similarity matrix. However, we believe that solutions should emerge from such experimentations that come and go between perceptive considerations and modelization.

\section{Acknowledgements}

Part of this work was conducted in the context of the European project CUIDADO. Thank you to Olivier Lartillot for the interesting discussions that we have had on this subject.

\section{References}

1. Peteers G et al, Toward Automatic Music Audio Summary Generation from Signal Analysis, Proc Ismir, Paris, 2002,

2. Lartillot O, Perception-Based Advanced Description of Abstract Musical Content, Proc. WIAMIS, London, 2003

3. Rolland P-Y, Discovering patterns in musical sequences, JNMR $28 \mathrm{~N}^{\circ} 4$ pp. 334-350, 1999 
4. Meredith et all, Algorithms for discovering repeated patterns in multidimensional representations of polyphonic music, Cambridge Music Processing Colloquium, 2003

5. Cambouropoulos E, Towards a General Computational Theory of Musical Structure, PhD, Edinburgh, Faculty of Music and Department of Artificial Intelligence, 1998

6. Meudic B, A causal algorithm for beat tracking, $2^{\text {nd }}$ conference on understanding and creating music, Caserta, Italy, 2002

7. Tversky A, Features of similarity, journal of Psychological Review, p327-352 1977 